\title{
Musculoskeletal ultrasound assessment in pediatric knee hypermobility: a case control study
}

\author{
Laura R. Ballenger ${ }^{1 *} \mathbb{D}$, Melissa Moore-Clingenpeel ${ }^{2}$ and Edward J. Oberle ${ }^{1}$
}

\begin{abstract}
Background: While musculoskeletal ultrasound (MSUS) use in pediatric rheumatology is becoming more common, the majority of pediatric MSUS literature continues to focus on ultrasound findings in healthy children and juvenile idiopathic arthritis with little discussion of other musculoskeletal problems that may mimic arthritis such as joint hypermobility. Chronic joint pain related to hypermobility is a common referral to pediatric rheumatology clinics. Our aim is to describe the musculoskeletal ultrasound (MSUS) characteristics of the knee in a population with joint hypermobility and pain in comparison to control participants.
\end{abstract}

Methods: Participants were recruited into three groups for a case-control study. Case group participants had knee hypermobility and pain symptoms $(H+P)$. Participants in one control group had knee hypermobility without pain symptoms (H-P), and participants in the other control group had no knee hypermobility or pain symptoms (NP). Bmode and Doppler MSUS images were obtained and scored for each knee. Descriptive statistics are used for demographic variables and MSUS findings. Regression analysis is used to evaluate risk of synovial effusion and higher synovial effusion/hypertrophy quantitative score.

Results: MSUS assessment was performed on 91 knees of 50 participants. $\mathrm{H}+\mathrm{P}$ knees were more likely to have positive findings noted on MSUS ( $94 \%$ vs. $70 \%$ of H-P and $74 \%$ of NP knees, $p=0.043$ ). Patellar tendon hyperemia was more common in $\mathrm{H}+\mathrm{P}$ knees (52\%, vs. $19 \%$ among $\mathrm{H}-\mathrm{P}$ and $23 \%$ among NP, $p=0.025)$. Participants who reported taking scheduled non-steroidal anti-inflammatory drugs (NSAIDs) had an increased risk of synovial effusion $(\mathrm{RR}=1.83,95 \% \mathrm{Cl}=1.07-2.30, p=0.026)$ and a trend towards increased risk of a higher synovial effusion/ hypertrophy quantitative score ( $\mathrm{RR}=1.77,95 \% \mathrm{Cl}=0.92-3.38, p=0.086)$.

Conclusions: While positive MSUS findings were frequent in all participants, patellar tendon hyperemia was more frequent in participants with knee hypermobility and pain symptoms. Additionally, reported use of NSAIDs was associated with an increased risk of synovial effusion and higher synovial effusion/hypertrophy quantitative score. Further study should assess correlation between tendon abnormalities and degree of pain symptoms as well as the effect of NSAIDs on MSUS findings.

Keywords: Ultrasonography, Hypermobility, Knee, Tendon hyperemia

\footnotetext{
*Correspondence: laura.ballenger@nationwidechildrens.org

'Department of Rheumatology, Nationwide Children's Hospital, 700

Children's Drive, Columbus, OH 43205, USA

Full list of author information is available at the end of the article
}

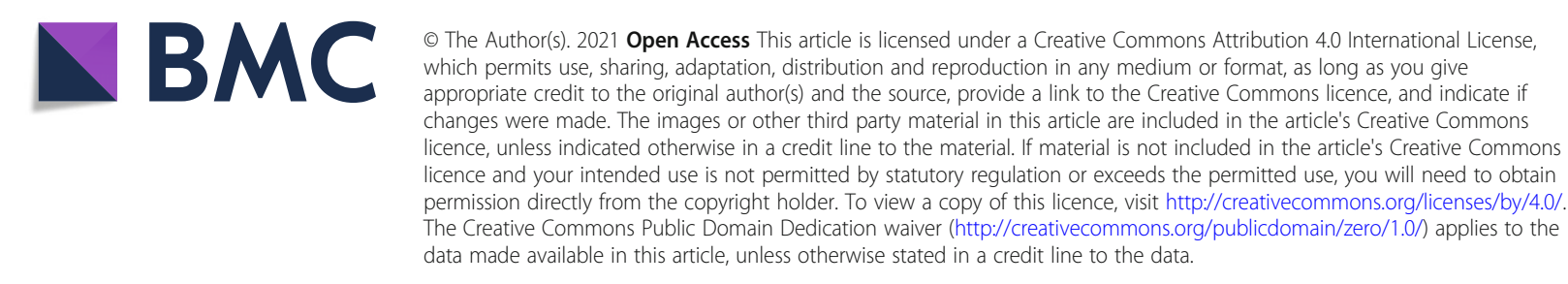




\section{Background}

Musculoskeletal pain is a common symptom in children and adolescents. The symptom becomes more prevalent as age increases [1] and is a frequent reason for evaluation in both primary and specialty care [2]. Joint hypermobility is a risk factor for musculoskeletal pain in adolescents, particularly in certain joints such as the knee [3, 4]. The prevalence of joint hypermobility varies in different populations but can affect over 30\% of children and adolescents [5]. The pathophysiology of joint hypermobility contributing to joint pain is unclear but may be related to excess movement leading to stress and micro-trauma [2]. Given the chronicity of the pain and the common delay in diagnosis of joint hypermobility [6, 7], children are often referred to pediatric rheumatology and other subspecialists for evaluation of joint pain.

Musculoskeletal ultrasound (MSUS) use is increasing within pediatric medicine, particularly within pediatric rheumatology $[8,9]$. MSUS is shown to be more sensitive than physical examination in detecting active synovitis and subclinical arthritis [10, 11]. Additionally, MSUS can reveal findings of tendon abnormalities and enthesitis $[12,13]$. To date, the majority of MSUS literature focuses on findings in healthy children and those with inflammatory arthritis, particularly juvenile idiopathic arthritis (JIA). Few studies exist regarding tendon measurements or elastography on ultrasound imaging in populations with joint hypermobility $[14,15]$. However, these studies include mostly adult participants and report minimal MSUS views of the joints.

It is important to describe the MSUS findings in joint hypermobility to potentially differentiate from other causes of chronic joint pain such as inflammatory arthritis, apophysitis, and tendinopathies. Specific abnormal findings from joint hypermobility could help with additional diagnostic or therapeutic decisions. Additionally, abnormal findings could provide further insight into the pathophysiology of pain associated with joint hypermobility.

Our aim is to describe the MSUS characteristics of the knee in a population with joint hypermobility and pain in comparison to asymptomatic children with and without joint hypermobility.

\section{Methods}

\section{Participant recruitment}

Participants were recruited and assigned by knee into three groups for a case-control study: case group with knee joint hypermobility and pain symptoms $(\mathrm{H}+\mathrm{P})$, control group with knee joint hypermobility without pain symptoms (H-P), and control group with no hypermobility or pain (NP). Participants in the case group were recruited from a pediatric rheumatology clinic with a multidisciplinary clinic dedicated to patients with joint hypermobility. Participants in the control groups were recruited from the same pediatric rheumatology clinic as well as a pediatric ophthalmology clinic and a pediatric dermatology clinic at an academic children's hospital. Ethics approval was obtained from the Nationwide Children's Institutional Review Board (IRB18-01176). Participants or their legal guardians if participants were under 18 years of age provided written informed consent.

Inclusion criteria for the case group were participant age $\geq 14$ years, $>10$ degrees of knee hyperextension on goniometer measurement per Beighton criteria for hypermobility [16], and pain score $\geq 1$ (out of 10) on validated visual pain scale [17] reported by the participant in each knee over the past month. Control participants were matched by age and gender. The H-P control group had knee hypermobility without pain symptoms (pain score $=0$ ). The NP control group had no knee hypermobility ( $\leq 10$ degrees of knee hyperextension) and no pain symptoms. A single physician performed goniometry of Beighton criteria for all participants. Participants in any group were excluded if they had a current diagnosis associated with arthritis, history of previous knee surgery, or history of knee trauma within the month prior to evaluation. Participant body mass index (BMI) and non-steroidal anti-inflammatory drug (NSAI D) use were collected from the medical record.

\section{Ultrasound assessment}

Participants had MSUS assessment of one or both knees, depending on their hypermobility measurements, pain symptoms and exclusion criteria. For example, if a participant had previous unilateral knee surgery, this knee was excluded, but they could enroll in the study for the opposite knee. A pediatric rheumatology fellow trained in MSUS obtained B-mode and Doppler images of each knee according to published guidelines, which include multiple views in the suprapatellar, infrapatellar, medial, lateral and posterior aspects of the knee while the knee is flexed slightly at 30 degrees [18]. MSUS were obtained with GE Logiq S8 equipment with a $4-12-\mathrm{MHz}$ linear array transducer. A blinded pediatric rheumatologist trained in MSUS scored the ultrasound images according to a pediatric scoring system with grading of zerothrough three-points on semi-quantitative scale for synovial effusion, hypertrophy and hyperemia [19]. On this scale, zero represents no findings while one through three represent grades of positive findings. The highest score seen on any view of the suprapatellar recess (midline anterior or medial/lateral gutters) of either an effusion or synovial hypertrophy was used as the grade for synovial effusion/hypertrophy quantitative score for each knee. Additionally, images were evaluated for presence 
or absence of tendon abnormalities, tendon thickness, and cartilage thickness based on previously published definitions [20-22].

\section{Statistical analysis}

Based on power analysis, 33 knees were needed per group for a total of 99 knees to achieve an 80\% power to detect a $28 \%$ difference in joint effusion rate between the groups, assuming a $60 \%$ rate of effusion among healthy knees [23, 24]. As these previous studies have reported variability in knee effusion rates from 60 to $80 \%$ in healthy knees, an effect size of greater than $20 \%$ was necessary to represent a clinically meaningful difference. Descriptive statistics are reported at the participant level for overall demographic variables and NSAID use. Descriptive statistics are reported at the knee level for all MSUS findings, pain scores, and any measurement that can vary between a participant's knees. Variables are compared by knee type using logistic, Poisson, and linear mixed effects models depending on the variable distribution. Comparisons are not made for variables with an event rate of less than five. Poisson generalized estimating equations with robust standards errors are used to evaluate the association of knee type with synovial effusion and synovial effusion/hypertrophy quantitative score while accounting for NSAID use and BMI. All analyses were conducted using SAS 9.4 (SAS Institute, Cary, NC).

\section{Results}

Fifty participants enrolled in the study, and MSUS was completed on 91 knees. Eight knees are missing from the control groups to achieve the desired study power as recruitment ended earlier than expected due to COVID-
19 pandemic and subsequent restrictions placed on research recruitment.

\section{Participant characteristics}

Participant characteristics are summarized in Table 1 by group. Three participants had one knee enrolled in the $\mathrm{H}+\mathrm{P}$ group and one knee enrolled in the $\mathrm{H}-\mathrm{P}$ group. Male participants accounted for about one-quarter of the population. BMI was not significantly different between the groups. NSAID use, either scheduled or as needed, was more common in participants of the $\mathrm{H}+\mathrm{P}$ knees $(63 \%$ vs. $18 \%$ of $\mathrm{H}-\mathrm{P}$ and $13 \%$ of NP participants, $p<0.001)$.

As expected, based on study design, NP knees had a significantly lower Beighton score compared to $\mathrm{H}+\mathrm{P}$ or $\mathrm{H}-\mathrm{P}$ knees, while there was no difference in Beighton score among $\mathrm{H}+\mathrm{P}$ and $\mathrm{H}-\mathrm{P}$ groups. Similarly, NP knees had less knee hyperextension by goniometry than $\mathrm{H}+\mathrm{P}$ or $\mathrm{H}$-P knees, while there was no difference among $\mathrm{H}+$ $\mathrm{P}$ and $\mathrm{H}-\mathrm{P}$ groups.

\section{Ultrasound findings}

MSUS findings are summarized in Table 2 by group. $\mathrm{H}+\mathrm{P}$ knees were more likely to have any type of positive finding noted on MSUS compared to control groups $(94 \%$ vs. $70 \%$ of H-P and $74 \%$ of NP knees, $p=0.043$ ), however, all three groups had a high percentage of positive findings. Over half of the knees in all groups had some degree of synovial effusion. Effusion was most common within the lateral parapatellar synovium in all three groups $(42 \%$ of $\mathrm{H}+\mathrm{P}, 33 \%$ of $\mathrm{H}-\mathrm{P}$, and $42 \%$ of NP knees) compared to the medial parapatellar $(33 \%$ of $\mathrm{H}+\mathrm{P}, 25 \%$ of $\mathrm{H}-\mathrm{P}$, and $32 \%$ of NP knees) and suprapatellar synovium (21\% of $\mathrm{H}+\mathrm{P}$, $19 \%$ of H-P, and $19 \%$ of NP knees). The synovial effusion/hypertrophy quantitative score did not differ

Table 1 Participant Characteristics

\begin{tabular}{|c|c|c|c|c|c|}
\hline Characteristics by participant & All $(n=50)$ & $\mathrm{H}+\mathrm{P}(n=20)$ & $\mathrm{H}-\mathrm{P}(n=16)$ & NP $(n=17)$ & $p$-value \\
\hline Male, n (\%) & $12(24)$ & $8(24)$ & $8(30)$ & $8(26)$ & 0.892 \\
\hline Age (years), mean (sd) & $16.1(1.4)$ & $16.2(1.2)$ & $16.0(1.4)$ & $16.2(1.5)$ & 0.764 \\
\hline BMl, mean (sd) & $26.1(6.7)$ & $26.7(6.9)$ & $26.0(5.5)$ & $25.5(7.5)$ & 0.762 \\
\hline Pain Score, mean (sd) & & $4.6(1.5)$ & & & \\
\hline Beighton Score, mean (sd) & $3.9(3.1)$ & $5.8(2.3)$ & $5.1(2.7)$ & $0.7(0.9)$ & $<0.001$ \\
\hline Knee measurement (degrees), mean (sd) & $189.6(4.9)$ & $192.9(1.9)$ & $192.7(1.8)$ & $183.3(2.3)$ & $<0.001$ \\
\hline Characteristics by knee & All $(n=91)$ & $\mathrm{H}+\mathrm{P}(n=33)$ & H-P $(n=27)$ & $\mathrm{NP}(n=31)$ & $p$-value \\
\hline NSAID use, n (\%) & & & & & $<0.001$ \\
\hline None & $61(67)$ & $12(36)$ & $22(81)$ & $27(87)$ & \\
\hline As needed & $18(13)$ & $11(33)$ & $3(11)$ & $4(13)$ & \\
\hline Scheduled & $12(13)$ & $10(30)$ & $2(7)$ & $0(0)$ & \\
\hline
\end{tabular}

Legend: Significant $p$-values appear in bold font. Three participants had one knee enrolled in the $\mathrm{H}+\mathrm{P}$ group and one knee enrolled in the $\mathrm{H}-\mathrm{P}$ group. $H+P$ hypermobility with pain group, $H-P$ hypermobility without pain group, NP no hypermobility or pain group, BMI body mass index, NSAID non-steroidal anti-inflammatory drug 
Table 2 Ultrasound Findings

\begin{tabular}{|c|c|c|c|c|c|}
\hline & All $(n=91)$ & $\mathrm{H}+\mathrm{P}(n=33)$ & $\mathrm{H}-\mathrm{P}(n=27)$ & NP $(n=31)$ & $p$-value \\
\hline Synovial Effusion, n (\%) & $52(57)$ & $21(64)$ & $14(52)$ & $17(55)$ & 0.714 \\
\hline Synovial Hypertrophy, n (\%) & $18(20)$ & $7(21)$ & $4(15)$ & $7(23)$ & 0.715 \\
\hline Synovial Effusion/Hypertrophy Quant Score ${ }^{a}$, mean (sd) & $1.1(0.9)$ & $1.1(0.9)$ & $0.8(0.7)$ & $1.0(1.0)$ & 0.488 \\
\hline Synovial Hyperemia, n (\%) & $2(2)$ & $1(3)$ & $1(4)$ & & \\
\hline Quadriceps Tendon Edema, n (\%) & $9(10)$ & $6(18)$ & $0(0)$ & $3(10)$ & 0.070 \\
\hline Quadriceps Tendon Hyperemia, n (\%) & $3(3)$ & $1(3)$ & $1(4)$ & $1(3)$ & 1.000 \\
\hline Quadriceps Tendon Tear, n (\%) & $1(1)$ & $1(3)$ & & & \\
\hline Quadriceps Tendon Enthesitis, n (\%) & $1(1)$ & $1(3)$ & & & \\
\hline Quad Tendon Width, Proximal, mm, mean (sd) & $5.1(1.0)$ & $5.0(0.9)$ & $5.0(0.7)$ & $5.5(1.1)$ & 0.144 \\
\hline Quad Tendon Width, Distal Insertion, mm, mean (sd) & $5.6(0.8)$ & $5.5(0.8)$ & $5.6(0.8)$ & $5.7(0.9)$ & 0.680 \\
\hline Patellar Tendon Edema, n (\%) & $4(4)$ & $3(9)$ & $1(3)$ & & \\
\hline Patellar Tendon Hyperemia, n (\%) & $29(32)$ & $17(52)$ & $5(19)$ & $7(23)$ & 0.025 \\
\hline Patellar Tendon Tear, n (\%) & $0(0)$ & & & & \\
\hline Patellar Tendon Enthesitis, n (\%) & $0(0)$ & & & & \\
\hline Patellar Tendon Width, Proximal Origin, mm, mean (sd) & $4.1(0.8)$ & $4.1(0.7)$ & $4.2(1.0)$ & $4.0(0.8)$ & 0.546 \\
\hline Patellar Tendon Width, Distal Insertion, mm, mean (sd) & $4.60(0.7)$ & $4.5(0.6)$ & $4.6(0.7)$ & $4.7(0.7)$ & 0.810 \\
\hline Pat Tendon Width, Smallest Central, mm, mean (sd) & $3.0(0.5)$ & $2.9(0.4)$ & $3(0.5)$ & $3.0(0.5)$ & 0.817 \\
\hline Cartilage Thickness, mm, mean (sd) & $2.6(0.7)$ & $2.9(0.7)$ & $2.4(0.5)$ & $2.6(0.7)$ & 0.414 \\
\hline Any Finding, n (\%) & $73(80)$ & $31(94)$ & $19(70)$ & $23(74)$ & 0.043 \\
\hline
\end{tabular}

Legend: Significant $p$-values appear in bold font. ${ }^{a}$ Synovial effusion/hypertrophy quantitative score is based on a zero to three-point Likert scale. $H+P$ hypermobility with pain group, $H-P$ hypermobility without pain group, $N P$ no hypermobility or pain group

among the groups (1.09 for $\mathrm{H}+\mathrm{P}, 0.78$ for $\mathrm{H}-\mathrm{P}$, and 0.97 for NP, $p=0.488$ ). Figure 1 shows representative examples from study participants of different synovial effusion/hypertrophy quantitative scores. The number of participants with quantitative score of two or greater on the scale of zero to three was similar amongst the groups $(24 \%$ of $\mathrm{H}+\mathrm{P}, 15 \%$ of $\mathrm{H}-\mathrm{P}$, and $27 \%$ of NP knees). Three knees in the NP group received a score of three for large effusion and/or significant synovial hypertrophy.

Patellar tendon hyperemia was a more common finding in $\mathrm{H}+\mathrm{P}$ knees $(52 \%$ vs. $19 \%$ of $\mathrm{H}-\mathrm{P}$ and $23 \%$

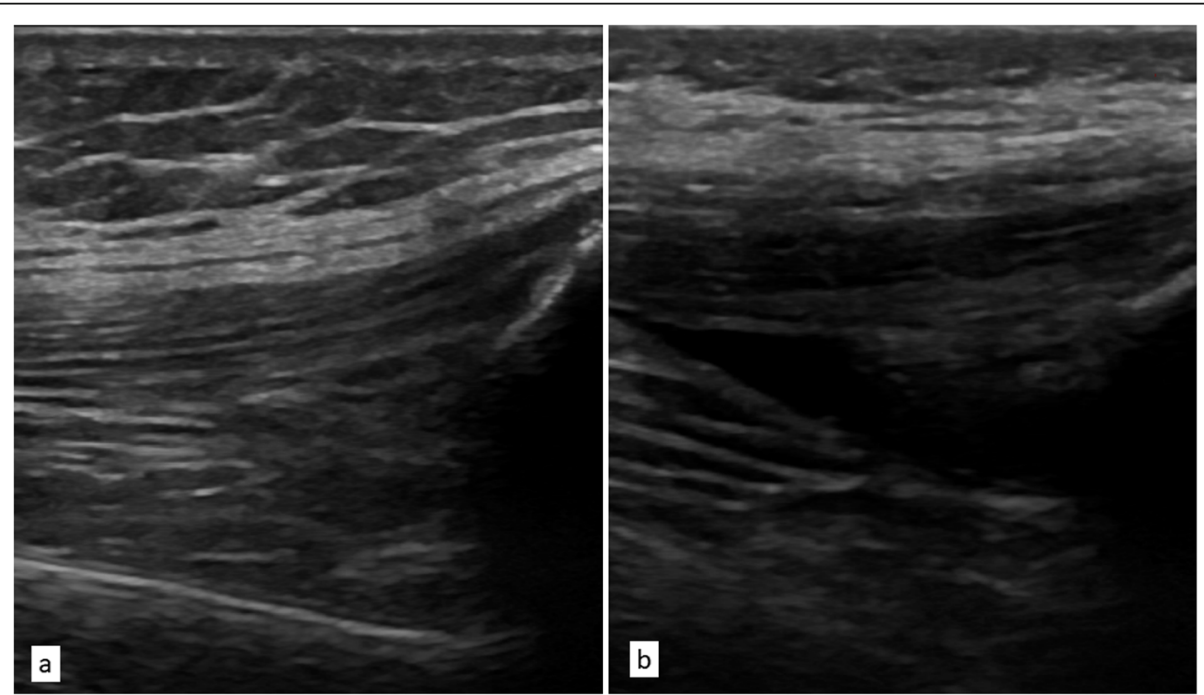

Fig. 1 Suprapatellar longitudinal images of participant knees in B-mode. Legend: a. Synovial effusion/hypertrophy quantitative score of zero. $\mathbf{b}$ Synovial effusion/hypertrophy quantitative score of two 
of NP knees, $p=0.025$ ). Figure 2 shows an example of positive findings in the patellar tendon of a study participant. Quadriceps tendon edema was also noted in more $\mathrm{H}+\mathrm{P}$ knees $(18 \%$ vs. $0 \%$ of $\mathrm{H}-\mathrm{P}$ and $10 \%$ of NP knees, $p=0.070$ ). Tendon thickness did not differ between the groups at any location for the quadriceps or patellar tendons. Cartilage thickness of the distal femur did not differ between the groups.

\section{Relative risk analyses}

Hypermobility, BMI, and NSAID use were evaluated as risk factors for any amount of synovial effusion (Table 3) and synovial effusion/hypertrophy quantitative score greater than zero (Table 4) in univariable and multivariable analyses. Hypermobile knees were not associated with an increased risk of effusion or synovial effusion/ hypertrophy quantitative score. Participants on scheduled NSAIDs had an $83 \%$ greater risk of effusion (adjusted relative risk $=1.83, p=0.026$ ) and $77 \%$ greater risk of a higher synovial effusion/hypertrophy quantitative score (adjusted relative risk $=1.77, p=0.086$ ).

\section{Discussion}

To the best of our knowledge, our study represents the first description of a comprehensive MSUS assessment of the knee in participants with joint hypermobility. The study identified several notable findings. Patellar tendon hyperemia was more common in the knees with hypermobility and pain symptoms. MSUS findings were frequent in all participants, however, participants with knee hypermobility and pain had significantly more positive findings. Synovial effusion was the most common finding in all three groups but was found with similar frequency amongst the groups. NSAID use was associated with an increased risk of synovial effusion and increased synovial effusion/hypertrophy quantitative score.

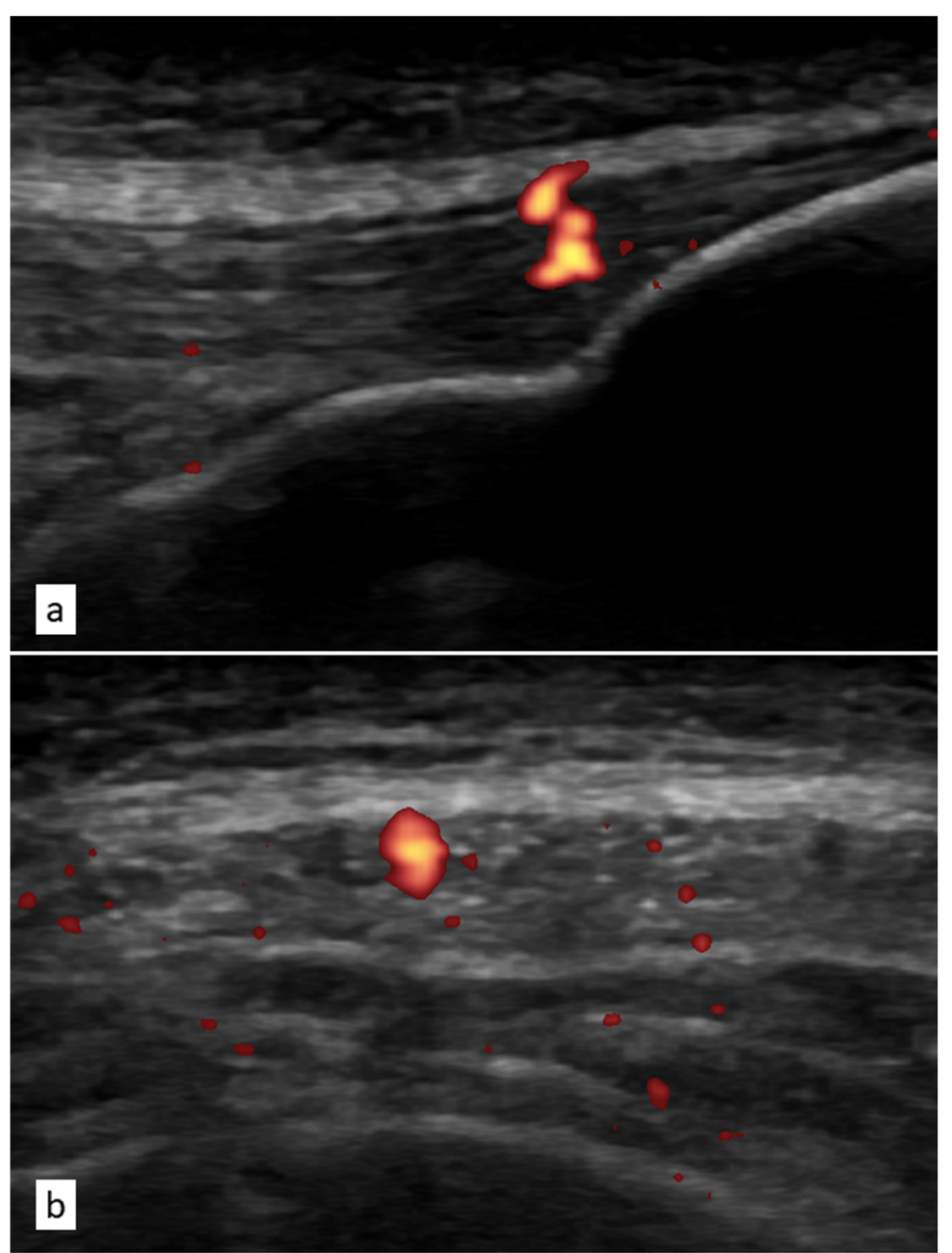

Fig. 2 Orthogonal infrapatellar images of the distal patellar tendon with positive Doppler findings. Legend: a. Longitudinal image. b. Transverse image 
Table 3 Risk of Any Synovial Effusion

\begin{tabular}{|c|c|c|c|c|c|c|}
\hline \multirow{2}{*}{ 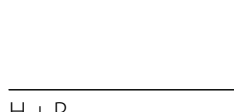 } & \multicolumn{3}{|c|}{ Univariate } & \multicolumn{3}{|c|}{ Multivariable } \\
\hline & $\mathrm{RR}$ & $95 \% \mathrm{Cl}$ & $p$-value & $\mathrm{RR}$ & $95 \% \mathrm{Cl}$ & $p$-value \\
\hline$H+P$ & 1.16 & $(0.73,1.85)$ & 0.534 & 0.91 & $(0.52,1.57)$ & 0.724 \\
\hline $\mathrm{H}-\mathrm{P}$ & 0.95 & $(0.54,1.65)$ & 0.844 & 0.90 & $(0.52,1.59)$ & 0.720 \\
\hline NP & reference & & reference & reference & & reference \\
\hline BMl & 1.00 & $(0.97,1.03)$ & 0.948 & 0.99 & $(0.96,1.02)$ & 0.431 \\
\hline No NSAIDs & reference & & reference & reference & & reference \\
\hline NSAIDs as needed & 1.20 & $(0.73,1.94)$ & 0.470 & 1.33 & $(0.76,2.30)$ & 0.317 \\
\hline Scheduled NSAIDs & 1.64 & $(1.14,2.36)$ & 0.008 & 1.83 & $(1.07,2.30)$ & 0.026 \\
\hline
\end{tabular}

Legend: Significant $p$-values appear in bold font. $H+P$ hypermobility with pain group, $H-P$ hypermobility without pain group, $N P$ no hypermobility or pain group, $B M I$ body mass index, NSAID non-steroidal anti-inflammatory drug

In previous reports of knee tendon features on MSUS in healthy children, no vascularity was detected in the patellar tendons $[25,26]$. Patellar tendon hyperemia is a significantly different finding in our case participants compared to control groups, which suggests that the tendon may be associated with pathology in hypermobile joints. Patellar tendon thickness at the proximal patellar ligament is similar in our whole study cohort to reports of tendon thickness in healthy children of similar ages ( $4.1 \mathrm{~mm}$ vs $3.5 \mathrm{~mm}$ [25] vs $4.0 \mathrm{~mm} \mathrm{[27]} \mathrm{at} \mathrm{the} \mathrm{proximal}$ patellar ligament). However, patellar tendon thickness at the distal patellar ligament is larger in our study compared to other reports in healthy children $(5.6 \mathrm{~mm}$ vs $3.5 \mathrm{~mm}$ [26] vs $3.7 \mathrm{~mm}$ [27]). Patellar tendon thickness was not significantly different between our case and control groups, so further investigation will be necessary to assess this finding of a difference in tendon thickness. Additionally, larger and longitudinal studies are necessary to further describe how these findings relate to degree of pain symptoms and how the findings change over time with symptoms.

The frequency of knee synovial effusion by MSUS in healthy children has been reported from 60 to $80 \%$ depending on age-group [23, 24]. In a German population of healthy children, the frequency of suprapatellar synovial effusions on MSUS in participants between ages 13 and 18 years was between 60 and 70\% [23], which is slightly higher than our percentage of control participants with synovial effusion in any space (suprapatellar, medial, lateral). Our percentage of case group participants with synovial effusion (64\%) is similar to the frequency of asymptomatic effusions in the German cohort. This similarity highlights that positive findings on MSUS do not necessarily correlate to underlying pathology. However, we investigated the medial and lateral parapatellar spaces in addition to the suprapatellar space which makes our study more sensitive for detecting any effusion. Additionally, the number of knees with higher synovial effusion/hypertrophy quantitative scores did not differ between the groups. It is likely that many of the participants in the $\mathrm{H}+\mathrm{P}$ case group have physiologic fluid on MSUS rather than abnormal or pathologic fluid that correlates with their pain symptoms. Three knees in the NP group received the highest quantitative score for synovial effusion/hypertrophy so the degree of positive findings was not able to differentiate the knees. The scoring tool [19] which was developed for patients with juvenile idiopathic arthritis may not be applicable to differentiate findings amongst this population.

Interestingly, use of NSAIDs was associated with an increased risk of synovial effusion and higher synovial effusion/hypertrophy quantitative score, even after

Table 4 Risk of Synovial Effusion/Hypertrophy Quantitative Score Greater Than Zero

\begin{tabular}{|c|c|c|c|c|c|c|}
\hline & \multicolumn{3}{|c|}{ Univariate } & \multicolumn{3}{|c|}{ Multivariable } \\
\hline & $\mathrm{RR}$ & $95 \% \mathrm{Cl}$ & $p$-value & RR & $95 \% \mathrm{Cl}$ & $p$-value \\
\hline $\mathrm{H}+\mathrm{P}$ & 1.13 & $(0.64,1.99)$ & 0.680 & 0.89 & $(0.42,1.87)$ & 0.753 \\
\hline $\mathrm{H}-\mathrm{P}$ & 0.80 & $(0.46,1.40)$ & 0.441 & 0.76 & $(0.44,1.33)$ & 0.337 \\
\hline NP & reference & & reference & reference & & reference \\
\hline BMI & 1.02 & $(0.99,1.05)$ & 0.292 & 1.01 & $(0.98,1.03)$ & 0.490 \\
\hline No NSAIDs & reference & & reference & reference & & reference \\
\hline NSAIDs as needed & 1.20 & $(0.64,2.24)$ & 0.575 & 1.13 & $(0.55,2.34)$ & 0.737 \\
\hline Scheduled NSAIDs & 1.79 & $(1,12,2.87)$ & 0.015 & 1.77 & $(0.92,3.38)$ & 0.086 \\
\hline
\end{tabular}

Legend: Significant $p$-value appears in bold font. $H+P$ hypermobility with pain group, $H$ - $P$ hypermobility without pain group, $N P$ no hypermobility or pain group, $B M I$ body mass index, NSAID non-steroidal anti-inflammatory drug 
accounting for participants' knee grouping. We suggest two potential explanations of this result as we would not expect for NSAIDs to increase synovial effusion or hypertrophy findings. NSAIDs are utilized as a therapy for inflammatory arthritis where there may be findings of synovial effusion, hypertrophy or hyperemia on MSUS [28], and these findings can be improved or masked with NSAID therapy [29]. In our study, NSAID use was significantly higher in participants from the case group. This medication may have been treating some of their MSUS findings, and if they were not taking the medication, more MSUS findings may have been present. Alternatively, scheduled NSAIDs may have partially treated symptoms and MSUS findings of an inflammatory arthritis. While none of the patients had received a diagnosis of inflammatory arthritis at the time of the study, the participants were not followed longitudinally after the study to see if their diagnosis changed over time. As a second potential explanation, the case group participants were recruited from a multi-disciplinary clinic for patients with joint hypermobility in which NSAIDs are a commonly prescribed medication for pain (per verbal discussion). If participants were recruited from primary or other specialty care clinics without this prescribing practice, the association of NSAID use and increased risk of synovial effusion and synovial effusion/hypertrophy quantitative score may not be significant. Lastly, the NSAID use variable was collected from review of the medical record. Participants may not have been taking NSAID as prescribed or may not have reported any over the counter NSAID use for documentation in the medical record. Future study should evaluate MSUS findings in comparison to actual NSAID use as well as potential subsequent diagnoses of inflammatory arthritis.

This study has several limitations. The number of participants is relatively low, and recruitment did not achieve the desired enrollment based on power calculations due to early recruitment conclusion for COVID-19 pandemic. A single pediatric rheumatology fellow obtained the MSUS images, and a single pediatric rheumatologist scored the images so these results may not be reproducible in an evaluation by multiple providers. However, the pediatric rheumatologist was blinded to the participant group and clinical information, and having a single reviewer eliminates potential for inter-rater variability. Additionally, all imaging parameters were scored with the knee flexed to 30 degrees, and while this is typical for assessment of joint space pathology there may have been some hyperemia of the tendons overlooked as this is best evaluated with the tendons maximally relaxed. Lastly, case participants were recruited from a single sub-specialty clinic so their findings may not be reflective of symptomatic knee hypermobility in the general population.

\section{Conclusions}

Our results suggest that the tendons may be an area of increased pathology in a population with knee hypermobility and pain symptoms. This population had frequent findings on MSUS, but all findings may not correlate with abnormal pathology as they also occur in participants without pain symptoms and without hypermobility findings. Positive findings occurred in many of the MSUS views which highlights the importance of a comprehensive MSUS assessment. Further study is needed to evaluate for MSUS findings and causation of pain symptoms.

\section{Abbreviations \\ BMI: Body mass index; $\mathrm{H}+\mathrm{P}$ : Hypermobility and pain symptoms; $\mathrm{H}-$ P: Hypermobility without pain symptoms; JIA: Juvenile idiopathic arthritis; MSUS: Musculoskeletal ultrasound; NP: No hypermobility or pain symptoms; NSAIDs: Non-steroidal anti-inflammatory drugs}

\section{Acknowledgments \\ None.}

Authors' contributions

LRB and EJO contributed to study conception, design, data collection and manuscript drafting. MMC contributed to statistical analysis. The authors read and approved the final manuscript.

\section{Author's information \\ None.}

\section{Funding}

This study was supported by an Intramural Funding Grant from the Research Institute at Nationwide Children's Hospital (FP00001977).

\section{Availability of data and materials}

The datasets used and/or analyzed during the current study are available from the corresponding author $[\mathrm{LRB}]$ on reasonable request. The data are not publicly available as this information could compromise the privacy of research participants.

\section{Declarations}

Ethics approval and consent to participate

Ethics approval was obtained from the Nationwide Children's Institutional Review Board (IRB18-01176). Participants or their legal guardians if participants were under 18 years of age provided written informed consent.

\section{Consent for publication}

Not applicable.

\section{Competing interests}

The authors declare that they have no competing interests.

\section{Author details}

${ }^{1}$ Department of Rheumatology, Nationwide Children's Hospital, 700 Children's Drive, Columbus, OH 43205, USA. ²Biostatistics Resource at Nationwide Children's Hospital, Abigail Wexner Research Institute, 700 Children's Drive, Columbus, OH 43205, USA.

Received: 19 October 2020 Accepted: 14 April 2021

Published online: 29 April 2021

\section{References}

1. De Inocencio J. Epidemiology of musculoskeletal pain in primary care. Arch Dis Child. 2004;89(5):431-4.

2. Cattalini M, Khubchandani R, Cimaz R. When flexibility is not necessarily a virtue: a review of hypermobility syndromes and chronic or 
recurrentmusculoskeletal pain in children. Pediatr Rheumatol Online J. 2015; 13(1):40. https://doi.org/10.1186/s12969-015-0039-3.

3. Tobias J, Deere K, Palmer S, Clark E, Clinch J. Hypermobility is a risk factor for musculoskeletal pain in adolescence: findings from a prospective cohort study. Rheumatology. 2013;52:33.

4. Sohrbeck-Nøhr O, Kristensen JH, Boyle E, Remvig L, Juul-Kristensen B. Generalized joint hypermobility in childhood is a possible risk for the development of joint pain in adolescence: a cohort study. BMC Pediatr. 2014;14:302. https://doi.org/10.1186/s12887-014-0302-7.

5. Scheper MC, Engelbert RH, Rameckers EA, Verbunt J, Remvig L, JuulKristensen B. Children with generalised joint hypermobility and musculoskeletal complaints: state of the art on diagnostics, clinical characteristics, and treatment. Biomed Res Int. 2013;2013:121054.

6. Adib N, Davies K, Grahame R, Woo P, Murray KJ. Joint hypermobility syndrome in childhood. A not so benign multisystem disorder? Rheumatology. 2005;44(6):744-50.

7. Murray KJ. Hypermobility disorders in children and adolescents. Best Pract Res Clin Rheumatol. 2006;20(2):329-51.

8. Nieto-Gonzalez JC, Monteagudo I, Vargas-Henny L, Janta I, Naredo E, Carreno L. Impact of musculoskeletal ultrasound on clinical practice in paediatric rheumatology. Clin Exp Rheumatol. 2015;33(4):583-7.

9. Magni-Manzoni S, Collado P, Jousse-Joulin S, Naredo E, D'Agostino M-A, Muratore V, et al. Current state of musculoskeletal ultrasound in paediatric rheumatology: results of an international survey. Rheumatology. 2014;53(3): 491-6.

10. Janow GL, Panghaal V, Trinh A, Badger D, Levin TL, llowite NT. Detection of active disease in juvenile idiopathic arthritis: sensitivity and specificity of the physical examination vs ultrasound. J Rheumatol. 2011;38(12):2671-4.

11. Magni-Manzoni S, Epis O, Ravelli A, Klersy C, Visconti C, Lanni S, et al. Comparison of clinical versus ultrasound-determined synovitis in juvenile idiopathic arthritis. Arthritis Rheumatism Arthritis Care Res. 2009;61 (11):1497504.

12. Weiss PF, Chauvin NA, Klink AJ, Localio R, Feudtner C, Jaramillo D, et al. Detection of Enthesitis in children with Enthesitis-related arthritis Dolorimetry compared to ultrasonography. Arthritis Rheumatol. 2014;66(1): 218-27.

13. Jousse-Joulin S, Breton S, Cangemi C, Fenoll B, Bressolette L, de Parscau L, et al. Ultrasonography for detecting Enthesitis in juvenile idiopathic arthritis. Arthritis Care Res. 2011;63(6):849-55.

14. Palmer S, Denner E, Riglar M, Scannell H, Webb S, Young G. Quantitative measures of tissue mechanics to detect hypermobile Ehlers-Danlos syndrome and hypermobility syndrome disorders: a systematic review. Clin Rheumatol. 2020;39(3):715-25.

15. Koçyiğit F, Kuyucu E, Koçyiğit A, Karabulut N. Real-time sonoelastography findings of a hypermobile child: a new technique in the assessment of tendon laxity. Rheumatol Int. 2015;35(12):2115-7.

16. Beighton P, Solomon L, Soskolne CL. Articular mobility in an African population. Ann Rheum Dis. 1973;32(5):413-8.

17. Wong DLBC. Pain in children: comparison of assessment scales. Pediatr Nurs. 1988;14(1):9-17.

18. Backhaus M, Burmester GR, Gerber T, Grassi W, Machold KP, Swen WA, et al. Guidelines for musculoskeletal ultrasound in rheumatology. Ann Rheum Dis. 2001:60(7):641-9.

19. Ting TV, Vega-Fernandez P, Oberle EJ, et al. Novel Ultrasound Image Acquisition Protocol and Scoring System for the Pediatric Knee. Arthritis Care Res (Hoboken). 2019;71(7):977-85. https://doi.org/10.1002/acr.23746.

20. Roth J, Ravagnani V, Backhaus M, Balint P, Bruns A, Bruyn GA, et al. Preliminary definitions for the sonographic features of synovitis in children. Arthritis Care Res (Hoboken). 2017;69(8):1217-23.

21. Wakefield RJ, Balint PV, Szkudlarek M, Filippucci E, Backhaus M, D'Agostino $M A$, et al. Musculoskeletal ultrasound including definitions for ultrasonographic pathology. J Rheumatol. 2005;32(12):2485-7.

22. Balint PV, Terslev L, Aegerter P, Bruyn GAW, Chary-Valckenaere I, Gandjbakhch F, et al. Reliability of a consensus-based ultrasound definition and scoring for enthesitis in spondyloarthritis and psoriatic arthritis: an OMERACT US initiative. Ann Rheum Dis. 2018;77(12):1730-5.

23. Windschall D, Trauzeddel R, Haller M, Krumrey-Langkammerer M, NimtzTalaska A, Berendes R, et al. Pediatric musculoskeletal ultrasound: age- and sex-related normal B-mode findings of the knee. Rheumatol Int. 2016;36(11): 1569-77.
24. Collado P, Naredo E, Calvo C, Crespo M. Assessment of the joint recesses and tendon sheaths in healthy children by high-resolution B-mode and power Doppler sonography. Clin Exp Rheumatol. 2007;25(6):915-21.

25. Chauvin NA, Ho-Fung V, Jaramillo D, Edgar JC, Weiss PF. Ultrasound of the joints and entheses in healthy children. Pediatr Radiol. 2015;45(9):1344-54.

26. Jousse-Joulin S, Cangemi C, Gerard S, Gestin S, Bressollette L, de Parscau L, et al. Normal sonoanatomy of the paediatric entheses including echostructure and vascularisation changes during growth. Eur Radiol. 2015; 25(7):2143-52.

27. Lin C, Diab M, Milojevic D. Grey-scale ultrasound findings of lower extremity entheses in healthy children. Pediatr Rheumatol Online J. 2015;13:14.

28. Roth J, Jousse-Joulin S, Magni-Manzoni S, Rodriguez A, Tzaribachev N, lagnocco A, et al. Definitions for the sonographic features of joints in healthy children. Arthritis Care Res (Hoboken). 2015;67(1):136-42.

29. Zayat AS, Conaghan PG, Sharif M, Freeston JE, Wenham C, Hensor EM, et al. Do non-steroidal anti-inflammatory drugs have a significant effect on detection and grading of ultrasound-detected synovitis in patients with rheumatoid arthritis? Results from a randomised study. Ann Rheum Dis. 2011;70(10):1746-51.

\section{Publisher's Note}

Springer Nature remains neutral with regard to jurisdictional claims in published maps and institutional affiliations.
Ready to submit your research? Choose BMC and benefit from:

- fast, convenient online submission

- thorough peer review by experienced researchers in your field

- rapid publication on acceptance

- support for research data, including large and complex data types

- gold Open Access which fosters wider collaboration and increased citations

- maximum visibility for your research: over $100 \mathrm{M}$ website views per year

At BMC, research is always in progress.

Learn more biomedcentral.com/submissions 